\title{
The Dortmund endoscopic full-thickness resection method: combination of the over-the-scope clip system and the Inoue cap
}
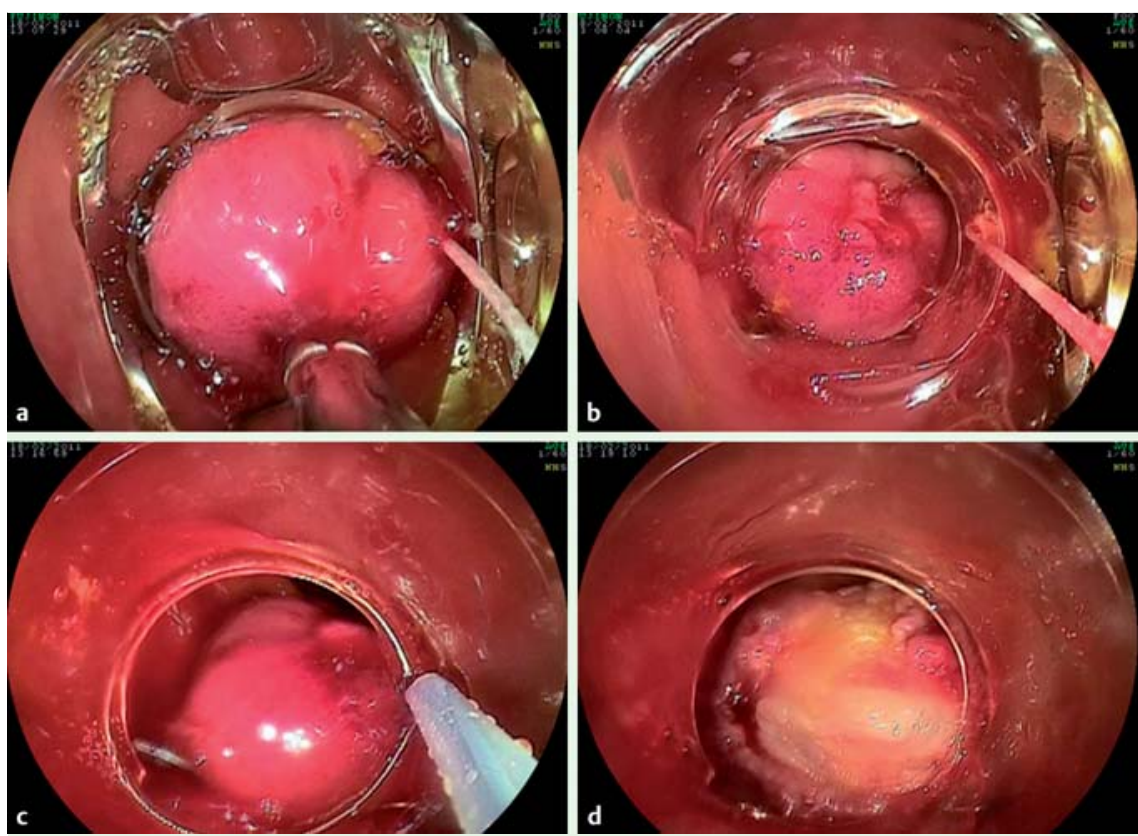

Fig. 1 Use of the Dortmund endoscopic full-thickness resection method, combining the over-thescope clip (OTSC) system and the Inoue cap, following incomplete endoscopic resection of a sigmoid colon polyp. a The tissue is pulled into the cap of the OTSC. $\mathbf{b}$ The OTSC is released at the base of the pseudopolyp. $\mathbf{c}$ The Inoue endoscopic mucosal resection kit (cap with snare) suctioning the pseudopolyp above the clip. $\mathbf{d}$ The bowel base after resection.

The over-the-scope clip (OTSC) system was developed for the closure of perforations and fistulas, and for the endoscopic therapy of gastrointestinal bleeding. Small mucosal and submucosal neoplastic lesions can be aspirated into the cap and, after releasing the clip, a "pseudopolyp" is created. This pseudopolyp located above the closed clip can then be resected using a polypectomy snare. However, placing a snare on the pseudopolyp may be difficult due to the shape of the clip.Herein, we present a dual technique using both the OTSC and the Inoue cap to accomplish endoscopic full-thickness resection (eFTR). An 80-year-old patient who had undergone incomplete endoscopic resection
(R1) of a 15-mm sigmoid colon polyp with carcinoma (SM1), underwent an eFTR of the remaining base of the lesion using this new technique. The procedure was performed 14 days after initial polypectomy. The steps of the technique were as follows. 1) The tissue was pulled into the cap of the OTSC system (14/6t; Ovesco Endoscopy AG, Tübingen, Germany) using a grasping forceps to create a pseudopolyp. An important aspect of this technique is to avoid fitting too much of the cap over the distal end of the endoscope, as it is important to ensure that there is sufficient cap volume for the entrapment of tissue. 2) The OTSC was then released at the base of the pseudopolyp, thus securing the bowel wall. 3) The scope was removed, and the Inoue endoscopic mucosal resection (EMR) cap (Olympus, Hamburg, Germany) was loaded onto the endoscope. 4) The Inoue cap loaded with the snare was then directed onto the pseudopolyp and a resection was performed above the OTSC ( Fig. 1). Of note, this part of the procedure is not a simple EMR, as the resulting resection often results in FTR. An additional advantage of this technique is that the distal web of the Inoue cap may function as electric insulation, thus preventing the contact of the resection snare with the exposed metallic clip.

The size of the resection specimen was $25 \mathrm{~mm}$. Histological analysis did not reveal any carcinoma in the lateral or vertical margins of the lesion. The patient recovered well and surgery was thus avoided.

Endoscopy_UCTN_Code_CPL_1AJ_2AD

Competing interests: None

\section{Martin Fähndrich, Michael Heike, Marcel Sandmann}

Interventional Endoscopy, Department of Gastroenterology, Klinikum Dortmund, Dortmund, Germany

\section{Bibliography}

DOI http://dx.doi.org/

$10.1055 / \mathrm{s}-0034-1377548$

Endoscopy 2014; 46: E466

(c) Georg Thieme Verlag KG

Stuttgart · New York

ISSN 0013-726X

Corresponding author

Martin Fähndrich, MD

Interventional Endoscopy

Department of Gastroenterology

Klinikum Dortmund

Beurhausstraße 40

44137 Dortmund

Germany

Fax: +49-231-95320510

martin.faehndrich@klinikumdo.de

martin.faehndrich@web.de 